Tous ces calculs, fastidieux par leur longueur, montrent donc en définitive que-l'intensité de la colostralisation est proportionnelle au rythme des accouchements, c'est-à-dire inversement proportionnelle au temps pendant lequel s'échelonnent les parturitions.

Plus cette durée est courte, plus les accouchements sont rapprochés, plus le lait de grand mélange contiendra de colostrum.

De même pour une période de vêlage déterminée, la proportion de colostrum est d'autant plus marquée que le nombre des vaches reproductrices est élevé.

On voit donc tout l'intérêt que l'on a de connaître ce qui se passe dans une zone de ramassage de lait : Nombre de vaches saillies, nombre de taureaux, dates des saillies, durée de l'échelonnement probable des parturitions, etc.

(A suivre.)

\title{
PRÉSENTATION D'APPAREIL
}

\section{UN NOUVEAU BUTYROMETTRE POUR L'APPLICATION DE LA IMÉTHODE GOTTLIEB-RÖSE}

\author{
par \\ JEAN PIEN \\ Ingénieur-Chimiste, Docteur ès Sciences \\ Directeur des Laboratoires de la Laiterie des Fermiers Réunis
}

On sait que la meilleure méthode de laboratoire pour le dosage de la matière grasse du lait est celle de Gottlieb-Röse qui utilise les principes suivants : la caséine est dissoute et la matière grasse désémulsionnée par un léger apport d'ammoniaque; les cénapses lipido-protidiques sont rompues par un apport d'alcool ; la matière grasse pure, libérée en totalité, est extraite par l'éther sulfurique en présence d'éther de pétrole.

Ce dosage peut s'effectuer dans n'importe quel récipient en forme de tube. Après agitation convenable, il se sépare deux couches : une couche hydroalcoolique sous-jacente renfermant en solution les éléments non gras du lait, une couche éthérée surnageante contenant toute la matière grasse. "

La difficulté consiste à prélever la totalité ou seulement une partie aliquote de la phase éthérée. Les tubes classiques de Rieter, de Röhrig ne permettent pas aisément de réaliser ce prélèvement avec sécurité et sans pertes.

Un premier grand progrès fut réalisé par la création du butyromètre Brioux constitué essentiellement par un tube gradué porteur de trois renflements et terminé par un bouchon émeri d'une conception spéciale (un peu semblable au bouchon des flacons compte- 
gouttes) : deux fentes diamétralement opposées peuvent coincider pour le vidage, l'une avec un bee d'évacuation du liquide, l'autre avec un petit orifice de rentrée d'air ; une rotation du bouchon de $90^{\circ}$ assure la fermeture totale. Le tube est légèrement coudé vers

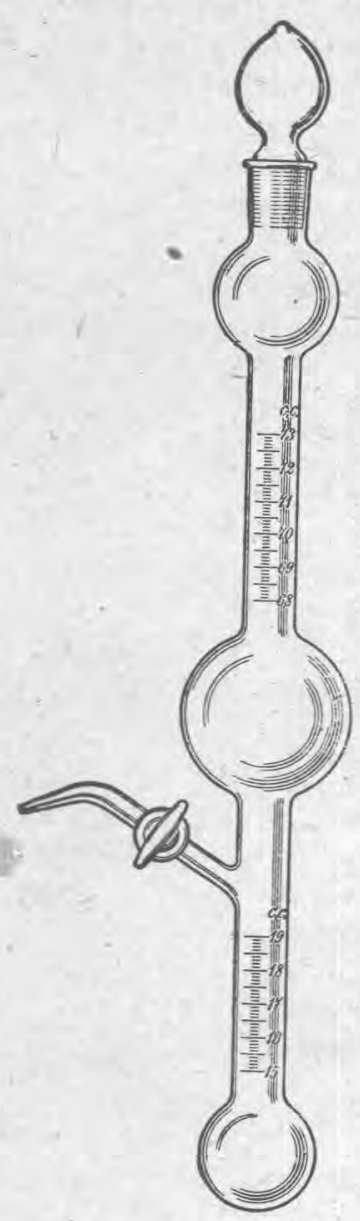
le milieu pour permettre, lors du vídage de l'éther par inclinaison du tube, de retenir la phase hydroalcoolique.

Cet appareil, que nous avons utilisé avec succès pendant de nombreuses années, présente néanmoins un inconvénient assez sérieux: l'opération du vidage de la phase surnageante est délicate; le tube doit être placé dans la position horizontale, ce qui risque d'amener la solution éthérée à sortir par l'orifice de rentrée d'air ; même sans aller jusque là, il peut arriver que par capillarité une petite quantité d'éther pénètre dans la fente de rentrée d'air du bouchon et arrête l'écoulement normal par l'orifice diamétralement opposé. En outre, si la longueur et la profondeur des fentes du bouchon ne sont pas exactement ce qu'elles doivent être, il en résulte un écoulement trop violent ou trop lent ou très difficile qui rend la manipulation malaisée. Enfin, les gouttes de solution éthérée qui restent dans le bec de vidage et dans les fentes du bouchon ne redescendent pas d'elles-mêmes dans l'appareil ; elles sont retenues par capillarité. Il est parfois difficile de les détacher et l'on court le risque d'avoir des pertes de matière.

Dans le triple but de parer à ces divers inconvénients, de rendre les manipulations plus aisées et de faire appel à un appareil moins onéreux, nous avons conçu un disposiṭ̣f légèrement différent que représente la figure ei-contre.

Dans ce nouveau butyromètre, basé exactement sur les mêmes principes, le vidage de la solution éthérée se fait par un ajutage latéral pourvu d'un robinet et situé vers la partie inférieure de la couche éthérée. La fermeture de la partie supérieure de l'appareil, est un bouchon émeri normal. Ce dispositif permet une extraction très facile et sans perte de la solution éthérée ; sa manipulation 
n'exige aucune précaution spéciale. Il est en outre moins onéreux que le butyromètre Brioux.

\section{Mode d'emploi}

10 Dans l'appareil propre et sec, introduire $10 \mathrm{~cm}^{3}$ de lait et $1-\mathrm{cm}^{3}$ d'ammoniaque à $22^{\circ}$ Bé. Agiter pendant une minute pour dissoudre la caséine et désémulsionner la matière grasse ;

$2^{\circ}$ Ajouter $10 \mathrm{~cm}^{3}$ d'alcool éthylique à $95^{\circ}$ et agiter de nouveau pendant une minute au moins. Pour réaliser cette agitation, boucher l'appareil dont le robinet latéral est fermé et amener les liquides dans la panse centrale ;

$3^{\circ}$ Ajouter $25 \mathrm{~cm}^{3}$ d'éther sulfurique. Boucher le butyromětre. Agiter violemment dans la position verticale et dans la position horizontale pendant au moins une minute ;

$4^{\circ}$ Laisser reposer quelques instants. Déboucher. Introduire. $25 \mathrm{~cm}^{3}$ d'éther de pétrole (D. $=0,650$ ). Boucher et agiter violemment comme ci-dessus pendant au moins une minute ;

$5^{\circ}$ Abandonner l'appareil dans la position verticale. Les deux couches se séparent rapidement. Le plan de démarcation s'établit entre les graduations 16 et 18 ;

$6^{\circ}$ Après un repos d'une demi-heure ou d'une heure, lire et noter les deux niveaux de la couche éthérée dont on déduit le volume total;

$7^{\circ}$ Déboucher l'appareil ; l'incliner à $45^{\circ}$ en plaçant l'ajutage latéral au-dessus d'un eristallisoir ou d'un ballon tarés, ouvrir le robinet et recueillir la liqueur éthérée. La forme de l'appareil permet de récolter aisément la majeure partie de cette solution éthérée sans risquer d'entraîner la liqueur sous-jacente, même pour une forte inclinaison de l'instrument;

$8^{\circ}$ En redressant le butyromètre, la petite quantité d'éther qui se trouvait dans l'ajutage latéral redescend dans le tube. Le niveau supérieur de la petite quantité restante de la solution éthérée se trouve à l'intérieur de la graduation;

$9^{\circ}$ Après un repos de quelques minutes, lire les deux niveaux de ce qui reste de solution éthérée et en déduire le volume;

$10^{\circ}$ Connaissant le volume total de la solution éthérée et le volume restant, on en déduit le volume prélevé. Après évaporation, séehage et pesée de la partie extraite, on déduit aisément le poids de matière grasse qui était renfermé dans la totalité de la couche éthérée c'est-à-dire dans $10 \mathrm{~cm}^{3}$ de lait (1).

(1) Nota. - L'appareil se prête évidemment bien à tous les dosages relevant de la méthode Gottlieb-Röse ou de la méthode. Schmidt-Bondzynski, en utilisant les techniques classiques dont l'application se trouve grandement facilitẻe par l'emploi de ce nouveau butyromètre.

(Constructeur : S. A. V. I. S., 20, rue de la Glacière, à Paris.) 